\title{
A Theory of Hygienic Macros
}

\author{
David Herman and Mitchell Wand \\ College of Computer and Information Science \\ Northeastern University \\ Boston, MA 02115 \\ $\{$ dherman, wand\}@ccs.neu. edu
}

\begin{abstract}
Hygienic macro systems, such as Scheme's, automatically rename variables to prevent unintentional variable capture - in short, they "just work." Yet hygiene has never been formally presented as a specification rather than an algorithm. According to folklore, the definition of hygienic macro expansion hinges on the preservation of alpha-equivalence. But the only known notion of alpha-equivalence for programs with macros depends on the results of macro expansion! We break this circularity by introducing explicit binding specifications into the syntax of macro definitions, permitting a definition of alpha-equivalence independent of expansion. We define a semantics for a first-order subset of Scheme-like macros and prove hygiene as a consequence of confluence.
\end{abstract}

The subject of macro hygiene is not at all decided, and more research is needed to precisely state what hygiene formally means and [precisely which] assurances it provides.

-Oleg Kiselyov [1]

\section{What Are Hygienic Macros?}

Programming languages with hygienic macros automatically rename variables to prevent subtle but common bugs arising from unintentional variable capturethe experience of the practical programmer is that hygienic macros "just work." Numerous macro expansion algorithms for Scheme have been developed over many years [23456], and the Scheme standard has included hygienic macros since $\mathrm{R}^{4} \mathrm{RS}$ [7].

Yet to date, a formal specification for hygiene has been an elusive goal. Intuitively, macro researchers have always understood hygiene to mean preserving $\alpha$-equivalence. In particular, performing an $\alpha$-conversion of a bound variable should not result in a macro expansion that accidentally captures the renamed variable. But this idea has never been made precise.

Why should such a simple idea be so hard to formalize? The problem is this: since the only known binding forms in Scheme are the core forms, the binding structure of a Scheme expression does not become apparent until after it has been fully expanded to core Scheme. Thus $\alpha$-equivalence is only well-defined for Scheme programs that have been fully expanded, with no remaining instances of

S. Drossopoulou (Ed.): ESOP 2008, LNCS 4960, pp. 48 62, 2008.

(C) Springer-Verlag Berlin Heidelberg 2008 
macros. So if the conventional wisdom is correct, the definition of hygienic macro expansion relies on $\alpha$-equivalence, but the definition of $\alpha$-equivalence relies on the results of macro expansion! This circularity is clearly paradoxical, and the definition of hygiene has consequently remained a mystery.

But in practice, well-behaved macros follow regular binding disciplines consistently, independent of their particular expansion. For example, Scheme's let construct can be macro-defined using lambda, yet programmers rely on knowing the binding structure of let without actually thinking about its expansion. If the semantics of macros only had access to this binding structure in such a way that we could reason formally about the scope of Scheme programs without resorting to operational reasoning about their expansion, we could cut the Gordian knot and specify both $\alpha$-equivalence and hygiene in an intuitive and precise way.

To put it more succinctly, we argue that the binding structure of a macro is a part of its interface. In this paper, we make that interface explicit as a type annotation. Our type system is novel but incorporates ideas both from the shape types of Culpepper and Felleisen [8] and nominal datatypes of Gabbay and Pitts 9. With the aid of these type annotations, we define a notion of $\alpha$ equivalence for Scheme programs with first-order macros, i.e., macros that do not expand into subsequent macro definitions, and prove hygiene as a consequence of confluence. We discuss higher-order macros as future work in Section 9.

The organization of this paper is as follows. The next section introduces $\lambda_{m}$, a Scheme-like language with typed macros. Section 3 defines the $\alpha$-equivalence relation for $\lambda_{m}$, and Section 4 introduces the macro type system. Section 5 defines the macro expansion semantics. The next two sections present the key correctness theorems: type soundness in Section [6 and hygiene in Section 7 . In Section 8 we present a front end for parsing S-expressions as $\lambda_{m}$ expressions. Section 9 concludes with a discussion of related and future work.

\section{$2 \quad \lambda_{m}$ : An Intermediate Language for Modeling Macros}

In Scheme, macro expansion transforms S-expressions into a small, fixed set of core forms which the underlying compiler or interpreter is designed to recognize. Expansion eliminates uses of macros by translating them according to their definitions, repeating this process recursively until there are no derived forms left to translate. Thus macro expansion consumes programs in surface syntax:

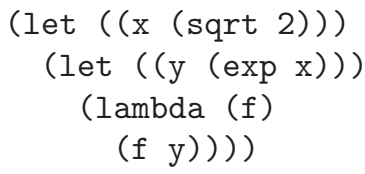

and produces programs with only the internal forms recognized by the compiler:

$$
((\lambda x \cdot((\lambda y \cdot \lambda f \cdot f y)(\exp x)))(\operatorname{sqrt} 2))
$$

We use a distinct syntax for core forms to highlight the fact that they indicate the completion of macro expansion. We use S-expressions not simply to describe 
Scheme, but as a simple and general model of tree-structured syntax. Because macro expansion operates on partially expanded programs, which may contain both core forms and S-expressions yet to be expanded, a model for macros must incorporate both syntactic elements.

To that end, we define an intermediate language for modeling macro expansion, called $\lambda_{m}$. The core forms are based on the $\lambda$-calculus, but with additional forms for local binding of macro definitions and macro application 1

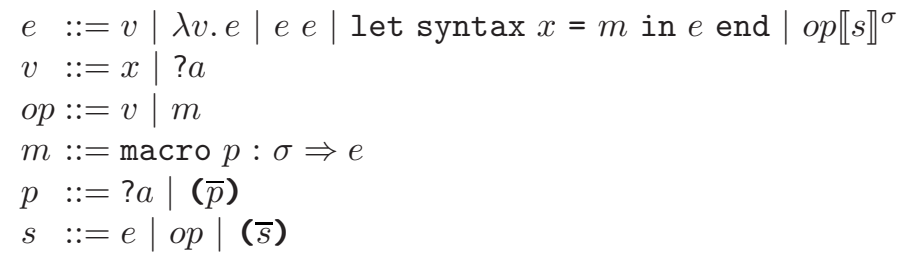

Unlike the surface syntax of Scheme, the syntax of $\lambda_{m}$ consists not just of Sexpressions but also expressions $e$, whose syntactic structure is fixed and manifest. Of course, macros admit arbitrary syntactic extension in the form of $\mathrm{S}$ expressions, so S-expressions $s$ appear in the grammar as the arguments to macro applications. Here too, though, the syntactic structure is made apparent via a shape type annotation $\sigma$. We return in detail to shape types in Section 2.2. Variables $v$ come in two sorts: program variables $x$, which are standard, and pattern variables ?a, which are bound in macro argument patterns and used in their definitions. Thus, for example, $\lambda x . x$ is a traditional $\lambda$-abstraction, but $\lambda ? a . ? a$ might appear in the body of a macro as a $\lambda$-abstraction whose bound variable will be provided from one of the macro's inputs. Macro operators op are either variable references or macro expressions. Macros $m$ contain a pattern $p$, a type annotation $\sigma$, and a template expression $e$. A pattern $p$ is a tree of pattern variables (assumed not to contain duplicates). Finally, an S-expression $s$ is a tree of expressions or macro operators. The latter form is used to pass macros as arguments to other macros.

The syntax of $\lambda_{m}$ may seem unfamiliar compared to the simple S-expressions of Scheme. After all, Scheme applications $(\bar{s})$ look different from $\lambda_{m}$ applications $o p \llbracket s \rrbracket^{\sigma}$ and in Scheme, pattern variables are indistinguishable from program variables. However, given shape-annotated macro definitions, we can easily parse surface S-expression syntax into $\lambda_{m}$. We describe this process in Section 8

\subsection{Tree Locations}

In order to address context-sensitive properties of terms, we use the mechanism of tree locations [10] to identify subterms by their position. Tree structures in our language take the general form $t::=L \mid(\bar{t})$ for some non-terminal of leaves $L$. For any such tree structure, we can select a subtree as a path from the root of the tree to the node containing the subtree. A tree location $\ell$ is an element of $\mathbb{N}^{*}$. Given a tree $t$, the subtree $t . \ell$ is defined by $t . \epsilon=t$ and $(\bar{t}) . i \ell=t_{i} . \ell$.

$\overline{1}$ Throughout this paper we use an overbar notation $(\bar{x})$ to represent sequences. 


\subsection{Binding Specifications}

Macro definitions and applications in $\lambda_{m}$ are explicitly annotated with shape types. The purpose of these annotations is to fix the structure of macros, including their scoping structure. For example, the following macro $m$ matches four pattern variables, ?a, ?b, ? $e_{1}$, and $? e_{2}$ :

$$
\begin{gathered}
\operatorname{macro}\left(? a ? b ? e_{1} ? e_{2}\right):\left(\langle 0\rangle\langle 1\rangle \mathbf{e x p r}^{0} \mathbf{e x p r}^{0,1}\right) \\
\quad \Rightarrow \lambda ? a .\left(\left(\lambda ? b . ? e_{2}\right) ? e_{1}\right)
\end{gathered}
$$

The shape type $\sigma=\left(\langle 0\rangle\langle 1\rangle\right.$ expr $^{0}$ expr $\left.^{0,1}\right)$ tells us that pattern variables $? a$ and $? b$ are placed in binding positions in the macro template, pattern variable $? e_{1}$ is used in the scope of $? a$ alone, and $? e_{1}$ appears inside the scope of both $? a$ and $? b$. Maintaining the bindings in order $-? a$ is bound outside, $? b$ inside - makes it possible to resolve references unambiguously even if both ?a and $? b$ are instantiated with the same variable. For example, this tells us that $m \llbracket\left(\begin{array}{llll}x & x & x & x\end{array}\right) \rrbracket^{\sigma}={ }_{\alpha} m \llbracket\left(\begin{array}{llll}x & y & x & y\end{array}\right) \rrbracket^{\sigma} \neq{ }_{\alpha} m \llbracket\left(\begin{array}{llll}x & y & y & x\end{array}\right) \rrbracket^{\sigma}$.

Shape types are defined by the following grammar:

$$
\begin{aligned}
& \tau::=\operatorname{expr} \mid \sigma \rightarrow \operatorname{expr} \\
& \beta::=\langle\ell\rangle \mid \operatorname{expr}_{\ell, \bar{\ell}} \\
& \sigma::=\tau|\beta|(\bar{\sigma})
\end{aligned}
$$

The base types $\tau$ include the type of expressions and the types of macros, which receive S-expressions as arguments and produce expressions. Binding types $\beta$ express the scope of S-expressions. A binder type $\langle\ell\rangle$ corresponds to a variable in binding position. The location $\ell$ represents the position in the macro S-expression where the binder occurs. A body type expr ${ }^{\ell, \bar{\ell}}$ corresponds to an expression inside the scope of one or more binders; the locations $\bar{\ell}$ indicate the positions in the macro S-expression of each of the binders that are in scope, in the order in which they are bound, outermost first.

\subsection{From S-Expressions to the Lambda Calculus}

Once a $\lambda_{m}$ program has been fully expanded, it consists only of core forms, which in our simple model corresponds to the untyped $\lambda$-calculus. We say a program is in expansion-normal form (ENF) if it obeys the familiar grammar:

$$
e::=x|\lambda x . e| e e
$$

If ENF is the internal language of the compiler or evaluator, then S-expressions are the surface language used by the programmer. The syntax of the surface language is a restricted subset of $\lambda_{m}$ S-expressions:

$$
s::=x \mid(\bar{s})
$$

Thus we can envision an idealized pipeline for the evaluation of programs with macros as shown in Figure 1 . 


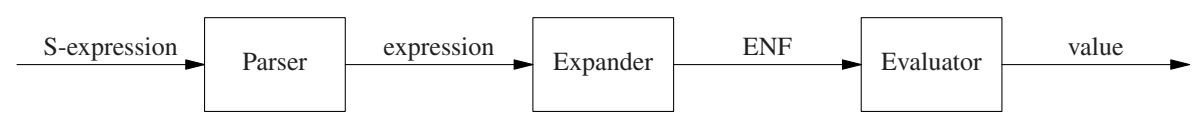

Fig. 1. Pipeline for an idealized evaluator with macro expansion

In real Scheme implementations, parsing is interleaved with macro expansion as the syntactic roles of expressions gradually become apparent. This is different from our idealized pipeline, which completely separates parsing from expansion. This is due to the fact that complete type information makes it possible to parse an S-expression before macro expansion. We return to the front end in Section 8 .

\section{Alpha-Equivalence}

We follow Gabbay and Pitts [9] in using variable swapping to define $\alpha$-equivalence. Swapping is defined by:

$$
\begin{aligned}
& \left(\begin{array}{ll}
v_{1} & v_{2}
\end{array}\right) \cdot v_{1}=v_{2} \\
& \left(\begin{array}{ll}
v_{1} & v_{2}
\end{array}\right) \cdot v_{2}=v_{1} \\
& \left(\begin{array}{ll}
\left.v_{1} v_{2}\right) \cdot v \quad=v & \text { if } v \notin\left\{v_{1}, v_{2}\right\}
\end{array}\right. \\
& \left(v_{1} v_{2}\right) \cdot \lambda v \cdot e=\lambda\left(\left(\begin{array}{ll}
v_{1} & v_{2}
\end{array}\right) \cdot v\right) \cdot\left(\left(\begin{array}{ll}
v_{1} & v_{2}
\end{array}\right) \cdot e\right) \\
& \left.\left(\begin{array}{ll}
v_{1} & v_{2}
\end{array}\right) \cdot(\bar{s})=\overline{\left(\left(v_{1} v_{2}\right) \cdot s\right.}\right) \\
& \text { etc. }
\end{aligned}
$$

The support of a term is the set of variables it contains:

$$
\begin{aligned}
& \operatorname{supp}(v)=\{v\} \\
& \operatorname{supp}(\lambda v \cdot e)=\{v\} \cup \operatorname{supp}(e) \\
& \operatorname{supp}((\bar{s}))=\bigcup_{i} \operatorname{supp}\left(s_{i}\right) \\
& \operatorname{etc.}
\end{aligned}
$$

A variable $v$ is fresh with respect to a finite set of terms $S$, written $v \# S$, if for all terms $s \in S, v \notin \operatorname{supp}(s)$. We write $v \# s_{1}, \ldots, s_{n}$ where $n \geq 1$ to mean $v \#\left\{s_{1}, \ldots, s_{n}\right\}$.

We also define the notion of simultaneously introducing multiple, distinct fresh variables by overloading the freshness relation for variable mappings. If $S$ is a set of terms and $Z$ is a mapping $\{\overline{\ell \mapsto z}\}$ then we write $Z \# S$ to mean

$$
\forall \ell \in \operatorname{dom}(Z) . Z(\ell) \# S \text { and } \forall \ell, \ell^{\prime} \in \operatorname{dom}(Z) . Z(\ell)=Z\left(\ell^{\prime}\right) \Rightarrow \ell=\ell^{\prime}
$$

We identify the binders of a form by collecting the set of binding positions identified in the form's shape type. The function $b p(\sigma)$ produces the set of binding positions of a shape type, and the function $p p(p)$ identifies the positions of pattern variables in a macro pattern.

$$
\begin{array}{llll}
b p(\mathbf{( \sigma )}) & =\bigcup_{i}\left\{i \ell \mid \ell \in b p\left(\sigma_{i}\right)\right\} & & p p(\mathbf{(} \overline{)})=\bigcup_{i}\left\{i \ell \mid \ell \in p p\left(p_{i}\right)\right\} \\
b p(\langle\ell\rangle) & =\{\epsilon\} & & p p(? a)=\{\epsilon\} \\
b p\left(\mathbf{e x p r}^{\ell}\right) & =b p(\tau)=\emptyset & &
\end{array}
$$


We can use $b p$ to compute the set of binders of a macro application $\operatorname{binders}(\sigma, s)$ as a mapping from binding positions $\ell$ to their actual binders $s . \ell$ :

$$
\operatorname{binders}(\sigma, s)=\{\ell \mapsto s . \ell \mid \ell \in b p(\sigma)\}
$$

\subsection{Shape-Directed Conversion}

Consider the following Scheme expression, with all occurrences of the variable $\mathrm{x}$ labelled for the sake of explanation.

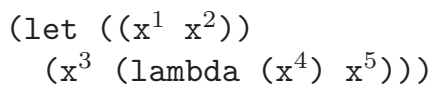

In order to $\alpha$-convert $\mathrm{x}^{1}$ to a fresh name $\mathrm{z}$, we must be careful to rename only the occurrences of $\mathrm{x}$ bound by $\mathrm{x}^{1}$, which in this example includes only $\mathrm{x}^{3}$. Because macros may have arbitrary shape, a structural induction on the S-expression would be insufficient to recognize which instances of $\mathrm{x}$ were which. Instead, we define a notion of shape-directed conversion $(Z X)^{\sigma} \cdot s$, which follows the structure of a form's binding specification rather than its syntax.

$$
\begin{aligned}
& (Z X)^{\tau} \quad \cdot s=s \\
& (Z X)^{\langle\ell\rangle} \cdot x=z \quad \text { if } z=Z(\ell) \\
& (Z X)^{\langle\ell\rangle} \cdot v=v \quad \text { if } \ell \notin \operatorname{dom}(Z) \\
& (Z X)^{\text {expr }^{\ell, \bar{\ell}^{\prime}}} \cdot e=(z x) \cdot(Z X)^{\text {expr }^{\overline{\ell^{\prime}}}} \cdot e \quad \text { if } z=Z(\ell) \text { and } x=X(\ell) \\
& (Z X)^{\text {expr }^{\ell, \overline{\ell^{\prime}}}} \cdot e=(Z X)^{\text {expr }^{\overline{\ell^{\prime}}}} \cdot e \quad \text { if } \ell \notin \operatorname{dom}(Z) \\
& (Z X)^{(\bar{\sigma})} \cdot(\bar{s})=\left(\overline{(Z X)^{\sigma_{i}} \cdot s_{i}}\right) \\
& (Z X)^{(\bar{\sigma})} \cdot ? a=? a
\end{aligned}
$$

The key to the definition of shape-directed conversion is the fourth rule, which swaps a bound variable with its corresponding fresh name in an expression within its scope. Because body types order their bound variables from the outside in, occurrences of the variable $x$ are renamed to $z$ only after performing all inner renamings, in case $x$ is shadowed by an inner binding.

\subsection{Alpha-Equivalence}

The definition of $\alpha$-equivalence appears in Figure 2. The first four rules parallel the rules of $\alpha$-equivalence for the $\lambda$-calculus, but note that we do not convert pattern variables $? a$ used in binding positions. The rule for macro bindings converts the macro name and proceeds inductively. The next rule is key: to compare two macro applications, their operators must be equivalent, and their arguments must be equivalent once we $\alpha$-convert their bound variables. Checking these involves several conditions. First, the two expressions must bind exactly the same pattern variables, if any; we ensure this by requiring that at any binding position $\ell, s . \ell$ binds an ordinary program variable $x$ if and only if $s^{\prime} . \ell$ binds an ordinary program variable $x^{\prime}$. We collect the binder mappings $X$ and $X^{\prime}$ for the 


$$
\begin{aligned}
& \overline{v={ }_{\alpha} v} \quad \frac{e={ }_{\alpha} e^{\prime}}{\lambda ? a \cdot e={ }_{\alpha} \lambda ? a \cdot e^{\prime}} \quad \frac{(z x) \cdot e==_{\alpha}\left(z x^{\prime}\right) \cdot e^{\prime}}{\lambda x \cdot e={ }_{\alpha} \lambda x^{\prime} \cdot e^{\prime}} \quad \begin{array}{c}
e_{1}={ }_{\alpha} e_{1}^{\prime} \\
e_{2}={ }_{\alpha} e_{2}^{\prime} \\
\hline e_{1} e_{2}={ }_{\alpha} e_{1}^{\prime} e_{2}^{\prime}
\end{array} \\
& z \# e, m, e^{\prime}, m^{\prime} \\
& \frac{m={ }_{\alpha} m^{\prime}(z x) \cdot e==_{\alpha}\left(z x^{\prime}\right) \cdot e^{\prime}}{\text { let } \operatorname{syntax} x=m \text { in } e \text { end }={ }_{\alpha} \text { let } \operatorname{syntax} x^{\prime}=m^{\prime} \text { in } e^{\prime} \text { end }} \\
& \begin{array}{c}
o p={ }_{\alpha} o p^{\prime} \\
\forall \ell \in b p(\sigma) \cdot \exists x=s \cdot \ell \Leftrightarrow \exists x^{\prime}=s^{\prime} \cdot \ell \\
X=\operatorname{binders}(\sigma, s) \quad X^{\prime}=\operatorname{binders}\left(\sigma, s^{\prime}\right) \\
Z=\{\ell \mapsto z \mid \ell \in b p(\sigma), \exists x=s \cdot \ell\} \quad Z \# s, s^{\prime} \\
\frac{(Z X)^{\sigma} \cdot s={ }_{\alpha}\left(Z X^{\prime}\right)^{\sigma} \cdot s^{\prime}}{o p \llbracket s \rrbracket^{\sigma}={ }_{\alpha} o p^{\prime} \llbracket s^{\prime} \rrbracket^{\sigma}}
\end{array} \\
& \forall \ell \in p p(p) \cdot p \cdot \ell=? a_{\ell} \text { and } p^{\prime} \cdot \ell=? a_{\ell}^{\prime} \text { and } ? z_{\ell} \# e, e^{\prime} \\
& \forall \ell, \ell^{\prime} \in p p(p) \cdot ? z_{\ell}=? z_{\ell^{\prime}} \Rightarrow \ell=\ell^{\prime} \\
& \left(\overline{? z_{\ell} ? a_{\ell}}\right) \cdot p=\left(\overline{? z_{\ell} ? a_{\ell}^{\prime}}\right) \cdot p^{\prime} \\
& \frac{\left(\overline{? z_{\ell} ? a_{\ell}}\right) \cdot e={ }_{\alpha}\left(\overline{? z_{\ell} ? a_{\ell}^{\prime}}\right) \cdot e^{\prime}}{\forall i . s_{i}={ }_{\alpha} s_{i}^{\prime}}
\end{aligned}
$$

Fig. 2. Alpha-equivalence of $\lambda_{m}$ programs

two respective forms, and we choose a mapping of fresh binders $Z$, being careful not to $\alpha$-convert at locations that bind pattern variables. Finally, we compare the $\alpha$-converted arguments $s$ and $s^{\prime}$. The rule for comparing macros is somewhat simpler. We choose fresh pattern variables $? z_{\ell}$ to replace the pattern variables in either macro, and compare both their patterns and templates. Finally, compound S-expressions are compared inductively.

\subsection{Instantiation}

Identifying binders in a shape type positionally is convenient for the theory, since it results in one canonical representation for each distinct type. However, for some operations it is necessary to identify binders by name. We present an alternate form of shape types $\hat{\sigma}$ which use variables rather than locations to represent their binding structure:

$$
\begin{aligned}
& \hat{\beta}::=\langle v\rangle \mid \operatorname{expr}^{v, \bar{v}} \\
& \hat{\sigma}::=\tau|\hat{\beta}|(\overline{\hat{\sigma}})
\end{aligned}
$$

We write $\hat{\sigma}=\sigma[X]$ to denote the instantiation of a nameless shape type $\sigma$ with the concrete variable names of a variable mapping $X$.

The free and bound variables of an expression are computed via shape-directed generalizations of the standard operations $F V(s, \hat{\sigma})$ and $B V(s, \hat{\sigma})$ (omitted for 
space). The following theorem ensures that we can always replace an S-expression with an $\alpha$-equivalent S-expression with fresh binders.

Theorem 1 (Freshness). Let $s$ be an $S$-expression and $S$ be a finite set of $S$ expressions. Then there exists an $S$-expression $s^{\prime}={ }_{\alpha} s$ such that $B V\left(s^{\prime}, \hat{\sigma}\right) \# S$.

Proof. Induction on the structure of $s$. For each binding in $s$, choose fresh binders that are not in $\operatorname{supp}(S)$.

It easy to show that if $e$ and $e^{\prime}$ are in ENF, then $e={ }_{\alpha} e^{\prime}$ if and only if the two expressions are $\alpha$-equivalent as $\lambda$-terms.

\section{Type Checking}

The job of the type checker is to confirm that each macro definition conforms to its specification and that each use of a macro conforms to its interface. Excerpts of the type checking algorithm are presented in Figure 3 . The type system uses two environments to track the two dimensions of binding in $\lambda_{m}$. The program environment $\Gamma::=\bullet \mid \Gamma[v:=\tau]$ tracks the scope of variables from binding forms such as $\lambda$ and let syntax. The pattern environment $\Phi \in\{\bullet\} \cup P$ Var $\rightarrow$ Shape tracks the binding of pattern variables for the current macro (if any). This environment is constructed by pairing the structure of a macro pattern $p$ with an instantiation of the macro's type annotation:

$$
\begin{aligned}
\operatorname{penv}(\mathbf{(} \bar{p}),(\overline{\hat{\sigma}})) & =\bigcup_{i} \operatorname{penv}\left(p_{i}, \hat{\sigma}_{i}\right) \\
\operatorname{penv}(? a, \hat{\sigma}) & =\{? a \mapsto \hat{\sigma}\}
\end{aligned}
$$

The type rule [T-MACDEF] permits only non-nested macro definitions by requiring an empty pattern environment. Rule [T-MACAPP] checks macro arguments with their annotated type instantiated with the actual binders. Rule [T-PBODY] checks a pattern variable reference with a body type, ensuring that all the necessary pattern variables have been bound in the proper order. Rule [T-PABs] checks abstractions with pattern variable binders. We discuss [T-PREF] in the next section. Rule [T-BODY] binds a variable from a body type in the program environment. Rule [T-MACRO] forms a pattern environment $\Phi$ and checks the template against its annotated type (subject to well-formedness constraints), filtering out any pattern variables from the program environment; the first-order macros of $\lambda_{m}$ cannot refer to pattern variables outside their own scope.

\subsection{The Aliasing Problem}

The design of our type system led us to discover a peculiarity of Scheme macros. Consider the following macro:

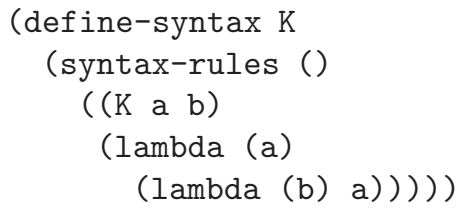




\section{$(\Gamma, \Phi) \vdash e: \mathbf{e x p r}$}

[T-MacDeF]

$$
\begin{gathered}
(\Gamma, \bullet) \vdash m: \sigma \rightarrow \mathbf{e x p r} \\
\frac{(\Gamma[m:=\sigma \rightarrow \mathbf{e x p r}], \bullet) \vdash e: \text { expr }}{(\Gamma, \bullet) \vdash \text { let } \operatorname{syntax} x=m \text { in } e \text { end }: \mathbf{e x p r}}
\end{gathered}
$$

[T-MACAPP]

$$
(\Gamma, \Phi) \vdash o p: \sigma \rightarrow \mathbf{e x p r}
$$$$
\frac{(\Gamma, \Phi) \vdash s: \sigma[\operatorname{binders}(\sigma, s)]}{(\Gamma, \Phi) \vdash o p \llbracket s \rrbracket^{\sigma}: \mathbf{e x p r}}
$$

[T-PBODY]

$\Phi(? a)=$ expr $^{\overline{? b}}$
$\frac{\left.\Gamma\right|_{\text {pvar }}=[? b:=\mathbf{e x p r}]}{(\Gamma, \Phi) \vdash ? a: \mathbf{e x p r}}$

$(\Gamma, \Phi) \vdash e: \hat{\beta}$
[T-PREF]

[T-PABS]

$\Phi(? a)=\langle ? a\rangle$

$\frac{(\Gamma[? a:=\mathbf{e x p r}], \Phi) \vdash e: \mathbf{e x p r}}{(\Gamma, \Phi) \vdash \lambda ? a . e: \mathbf{e x p r}}$

$$
\Phi(? a)=\langle ? a\rangle
$$

$\frac{\left.\Gamma\right|_{p v a r}=\Gamma^{\prime}[? a:=\mathbf{e x p r}]}{(\Gamma, \Phi) \vdash ? a: \mathbf{e x p r}}$

$(\Gamma, \Phi) \vdash o p: \sigma \rightarrow \mathbf{e x p r}$
[T-BoDy]

$$
\frac{(\Gamma[v:=\mathbf{e x p r}], \Phi) \vdash e: \mathbf{e x p r}^{\overline{v^{\prime}}}}{(\Gamma, \Phi) \vdash e: \operatorname{expr}^{v, \overline{v^{\prime}}}}
$$

[T-MACRO]

$$
\frac{\left(\left.\Gamma\right|_{v a r}, \operatorname{penv}(p, \sigma[\operatorname{pvars}(p)])\right) \vdash e: \mathbf{e x p r}}{(\Gamma, \Phi) \vdash(\operatorname{macro} p: \sigma \Rightarrow e): \sigma \rightarrow \mathbf{e x p r}}
$$

Fig. 3. Excerpts from the $\lambda_{m}$ type system

One might expect that any application of $\mathrm{K}$ would produce an expression equivalent to $\lambda x . \lambda y . x$. But consider the application $(\mathrm{K} \mathrm{x} \mathrm{x})$ : even in a hygienic macro system, this would expand into $\lambda x . \lambda x . x$ ! The binding structure of $\mathrm{K}$ is thus dependent on its actual arguments. We call this dependency the aliasing problem.

To resolve this ambiguity, we propose a simple rule we call the shadow restriction, enforced by the type rule [T-PREF]. A pattern binder ?a (i.e., of type $\langle ? a\rangle$ ) may only occur in an expression position if no other intervening pattern binders are in scope. For example, $\lambda ? a .(\lambda ? b . ? b)$ is legal but $\lambda ? a .(\lambda ? b . ? a)$ is ill-typed. In particular, this prohibits the definition of the $\mathrm{K}$ macro above. This restriction might seem draconian, but in fact $\mathrm{K}$ can easily be rewritten:

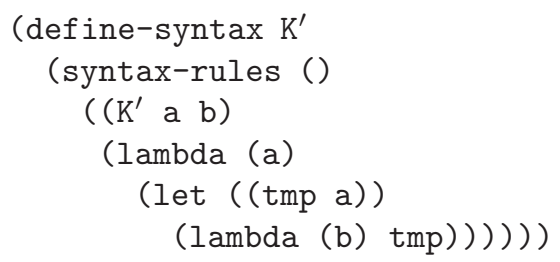

Note that even with standard, untyped Scheme macros, this new definition always exhibits the intended behavior, in that even $\left(K^{\prime} \mathrm{x} x\right)$ expands into an expression equivalent to $\lambda x . \lambda y . x$. 


\subsection{Alpha-Equivalence Preserves Type}

Theorem 2 gives us the freedom to use $\alpha$-equivalent $\mathrm{S}$-expressions without affecting the types.

Lemma 1. $(\Gamma, \Phi) \vdash s: \sigma[X] \Leftrightarrow(\Gamma, \Phi) \vdash(Z X)^{\sigma} \cdot s: \sigma[Z]$

Theorem 2 (Alpha-equivalence preserves type). If $(\Gamma, \Phi) \vdash s: \hat{\sigma}$ and $s={ }_{\alpha} s^{\prime}$ then $(\Gamma, \Phi) \vdash s^{\prime}: \hat{\sigma}$.

\section{Macro Expansion}

In this section, we specify our macro expansion semantics. We begin with a notion of compatibility, defined via expansion contexts.

\subsection{Expansion Contexts}

An expansion context $C^{\sigma}$ is an S-expression with a hole [], which produces an S-expression of shape $\sigma$ when filled with an expression $e$. When the shape of a context is clear or irrelevant, we omit it for brevity.

$$
\begin{aligned}
& C^{\operatorname{expr} \bar{\ell}^{\overline{2}}}::=[]\left|\lambda v \cdot C^{\mathbf{e x p r}}\right| C^{\text {expr }} e \mid e C^{\text {expr }} \\
& \text { let syntax } x=C^{\sigma \rightarrow \operatorname{expr}} \text { in } e \text { end } \\
& \text { let syntax } x=m \text { in } C^{\text {expr }} \text { end } \\
& C^{\sigma \rightarrow \operatorname{expr}}(s) \mid o p \llbracket C^{\sigma} \rrbracket^{\sigma} \\
& C^{(\bar{\sigma})} \quad::=\left(\bar{s}^{1 . . i-1} C^{\sigma_{i}} \bar{s}^{i+1 . .|\bar{\sigma}|}\right) \quad i \in 1 . .|\bar{\sigma}| \\
& C^{\sigma \rightarrow \operatorname{expr}}::=\operatorname{macro} p: \sigma \Rightarrow C^{\operatorname{expr}}
\end{aligned}
$$

\subsection{Variable Conventions}

The heart of hygienic macro expansion is the management of bindings to prevent accidental capture. Different expansion algorithms achieve this in different ways. For the specification of hygienic macro expansion, we simply specify the necessary conditions on variables under which expansion can proceed.

Analogous to the Barendregt variable convention [11, the transparent predicate allows a macro definition to be substituted into an application only if no intervening bindings can capture free variable references in the macro template. This condition is sometimes referred to as referential transparency.

$$
\operatorname{transparent}\left(s, \hat{\sigma}, s^{\prime}, \hat{\sigma}^{\prime}\right) \Leftrightarrow B V(s, \hat{\sigma}) \cap F V\left(s^{\prime}, \hat{\sigma}^{\prime}\right)=\emptyset
$$

This condition alone is not enough to prevent unintended capture. The predicate hygienic requires a macro template's bindings to be fresh before performing an application. This prevents the bindings in the template from capturing references in the macro's arguments.

$$
\operatorname{hygienic}\left(s, \hat{\sigma}, s^{\prime}\right) \Leftrightarrow B V(s, \hat{\sigma}) \# s^{\prime}
$$




\subsection{Expansion Semantics}

The semantics of macro expansion involves two rules. The first rule connects macro applications to their definitions via the substitution operation $s[x:=m]^{\hat{\sigma}}$, which uses the shape type $\hat{\sigma}$ to traverse the structure of $s$.

$$
\begin{array}{llrl}
v[x:=m]^{\text {expr }} & =v & (v \neq x) \\
(\lambda x . e)[x:=m]^{\text {expr }} & =\lambda x \cdot e & \\
(\lambda v \cdot e)[x:=m]^{\text {expr }} & =\lambda v \cdot\left(e[x:=m]^{\text {expr }}\right) & & (v \neq x) \\
x[x:=m]^{\sigma \rightarrow \operatorname{expr}} & =m & & \\
v[x:=m]^{\sigma \rightarrow \operatorname{expr}} & =v & & (v \neq x) \\
e[x:=m]^{\operatorname{expr}^{x, \bar{v}}} & =e & \\
e[x:=m]^{\operatorname{expr}^{v}, \bar{v}^{\prime}} & =e[x:=m]^{\text {expr }^{\bar{v}^{\prime}}} & & (v \neq x) \\
\text { etc. } & &
\end{array}
$$

A macro substitution step is defined by the rule:

$$
\begin{aligned}
& \text { let } \operatorname{syntax} x=m \text { in } e \text { end } \longmapsto \text { subst } e[x:=m]^{\text {expr }} \\
& \quad \text { if } \operatorname{transparent}(e, \text { expr, } m, \operatorname{typ} e(m))
\end{aligned}
$$

Note that the variable convention must be fulfilled to prevent the context of the macro application from capturing free variable references in the macro template.

The second rule of macro expansion performs a macro transcription step, expanding an individual macro application. This rule is carried out in two parts. The first part, pattern matching, matches the macro pattern against the actual sub-expressions, producing a substitution $\rho$ :

$$
\begin{aligned}
& \operatorname{match}\left(\mathbf{( p )}, \mathbf{( \overline { s } ) )}=\bigcup_{i} \operatorname{match}\left(p_{i}, s_{i}\right)\right. \\
& \operatorname{match}(? a, s)=\{? a \mapsto s\}
\end{aligned}
$$

Next, transcription instantiates all pattern variables in the template with the substitution function $\rho$ :

$$
\begin{array}{ll}
\operatorname{transcribe}(x, \rho) & =x \\
\operatorname{transcribe}(? a, \rho) & =\rho(? a) \\
\operatorname{transcribe}(\lambda v . e, \rho) & =\lambda(\operatorname{transcribe}(v, \rho)) \cdot(\operatorname{transcribe}(e, \rho)) \\
\operatorname{transcribe}\left(e_{1} e_{2}, \rho\right) & =\left(\operatorname{transcribe}\left(e_{1}, \rho\right)\right)\left(\operatorname{transcribe}\left(e_{2}, \rho\right)\right) \\
\operatorname{transcribe}\left(o p \llbracket s \rrbracket^{\sigma}, \rho\right) & =(\operatorname{transcribe}(o p, \rho)) \llbracket \operatorname{transcribe}(s, \rho) \rrbracket^{\sigma} \\
\operatorname{transcribe}(m, \rho) & =m \\
\operatorname{transcribe}(\mathbf{(}), \rho) & =\overline{(\operatorname{transcribe}(s, \rho))})
\end{array}
$$

The macro transcription step is defined as the rule:

$$
\begin{gathered}
\text { (macro } p: \sigma \Rightarrow e) \llbracket s \rrbracket^{\sigma} \longmapsto \text { trans } \operatorname{transcribe}(e, \operatorname{match}(p, s)) \\
\text { if transparent }(s, \hat{\sigma}, e, \mathbf{e x p r}) \text { and hygienic }(e, \mathbf{e x p r}, s) \\
\text { where } \hat{\sigma}=\sigma[\operatorname{binders}(\sigma, s)]
\end{gathered}
$$

The first variable convention also applies to this rule, since binders introduced in the actual arguments of the macro application should not capture free references 
from the template. The second convention prevents binders introduced from the body of the template from capturing references in the actual arguments.

We define the binary relation $\longmapsto_{\varepsilon}$ to be the compatible closure of the combined rules $\longmapsto_{\text {subst }} \cup \longmapsto$ trans on S-expressions up to $\alpha$-equivalence, i.e., the least relation such that $s_{1} \longmapsto s_{2}$ if there exist S-expressions $s_{1}^{\prime}, s_{2}^{\prime}$, a context $C$, and expressions $e_{1}, e_{2}$ such that $s_{1}={ }_{\alpha} s_{1}^{\prime}, s_{2}={ }_{\alpha} s_{2}^{\prime}, s_{1}^{\prime}=C\left[e_{1}\right], s_{2}^{\prime}=C\left[e_{2}\right]$, and either $e_{1} \longmapsto$ subst $e_{2}$ or $e_{1} \longmapsto$ trans $e_{2}$.

The binary relation $\longmapsto_{\varepsilon}$ is the reflexive, transitive closure of $\longmapsto_{\varepsilon}$.

\section{Type Soundness}

The type soundness proof is in the style of Wright and Felleisen [12]. The Preservation Lemma is proved for any S-expression $s$; it is reused in this more general form for the proof of confluence.

Lemma 2 (Preservation). If $(\Gamma, \Phi) \vdash s: \hat{\sigma}$ and $s \longmapsto \varepsilon s^{\prime}$ then $(\Gamma, \Phi) \vdash s^{\prime}: \hat{\sigma}$.

Proof. The proof depends on three lemmas that guarantee that macro substitution, pattern matching, and transcription respectively preserve type, as well as a decomposition lemma. Theorem 2 ensures that choosing $\alpha$-equivalent terms to satisfy the variable conventions is also type-preserving.

Lemma 3 (Progress). If $\vdash e$ : expr then either $e$ is in ENF or there exists an $e^{\prime}$ such that $e \longmapsto \varepsilon e^{\prime}$.

Proof. Macro substitution is defined for all well-typed S-expressions, as is match. Theorem 1 allows us to choose $\alpha$-equivalent terms that satisfy the variable conventions for the expansion rules.

Theorem 3 (Type soundness). If $\vdash e$ : expr and $e \longmapsto_{\varepsilon} e^{\prime}$ and $e^{\prime} \longmapsto_{\varepsilon}$, then $e^{\prime}$ is in ENF and $\vdash e^{\prime}$ : expr.

\section{$7 \quad$ Hygiene}

Theorem 4 (Confluence). Let $s$ be an $S$-expression such that $(\Gamma, \Phi) \vdash s: \hat{\sigma}$.

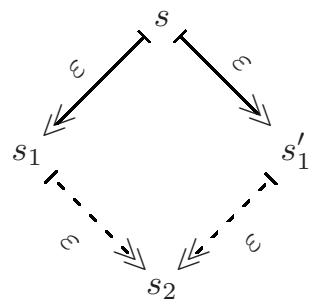

Proof. In the style of Barendregt [11, Chapter 11, §1. The proof involves marking a redex and tracking the marked redex and any copies or expansions of that marked term through multiple expansion steps. The central lemma shows that both macro substitution and transcription commute with expansion of marked redexes. 
At last, the final Hygiene Theorem follows immediately from confluence.

Theorem 5 (Hygiene). Let $e_{0}$ be an expression such that $\vdash e_{0}$ : expr. If $e_{0}={ }_{\alpha} e_{0}^{\prime}, e_{0} \longmapsto \varepsilon e$, and $e_{0}^{\prime} \longmapsto \varepsilon e^{\prime}$ such that $e$ and $e^{\prime}$ are in ENF, then $e={ }_{\alpha} e^{\prime}$.

This theorem provides the crucial guarantee of hygienic macros, namely that $\alpha$-conversion of $\lambda_{m}$ programs is semantics-preserving.

\section{Front End}

The parsing algorithm uses the same environments as the type system in order to distinguish the sorts of variables as well as annotate macro applications with types. Excerpts of this parsing algorithm are presented in Figure 4. Because function application in Scheme is denoted by parenthesization rather than invoking a special application macro, the rule for parsing function applications inserts an explicit reference to a built-in macro @. This is similar to the technique used in PLT Scheme [13, in which implicit function applications are rewritten to explicit applications of \#\%app.

Scheme implementations generally provide a standard library of macros. The primitive forms lambda and @ can be implemented as built-in macros in the initial context of a Scheme program:

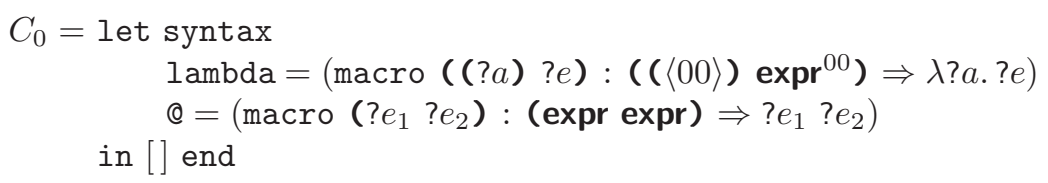

The parser must account for these macros in its initial environment:

$$
\begin{aligned}
& \Gamma_{0}(\text { lambda })=\left(\left((\langle 00\rangle) \text { expr }^{00}\right) \rightarrow\right. \text { expr } \\
& \Gamma_{0}(@)=(\text { expr expr }) \rightarrow \text { expr }
\end{aligned}
$$

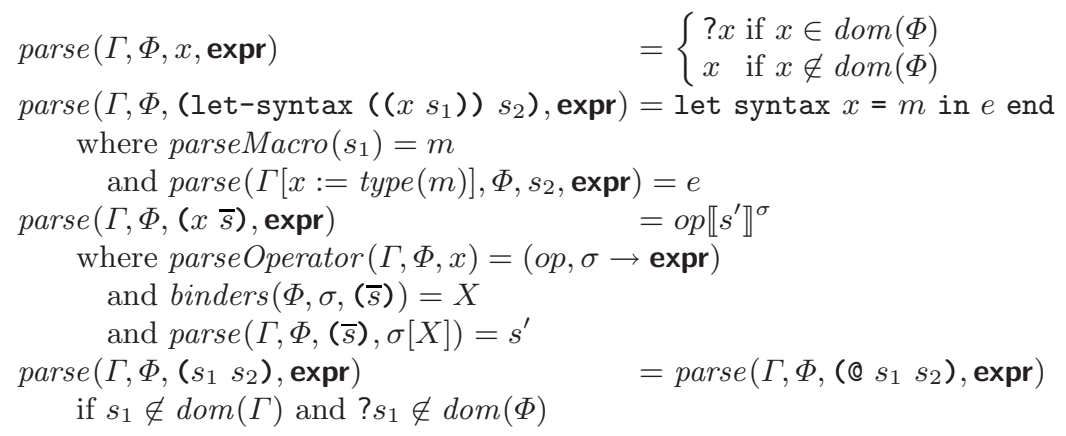

Fig. 4. Excerpts of the type-directed parsing algorithm 


\section{Related and Future Work}

Hygienic macros are over twenty years old, and many macro systems have been designed to facilitate or guarantee hygiene [2536]. Several have been defined in a rigorous and formal way, but none provides a specification for hygiene, nor any satisfying account for the guarantees it provides. Our work shares a common observation with the syntactic closures macro system 4], namely that macro programmers know the binding structure of macros a priori; their work provides an API rather than a theory. Our primitive lambda and @ macros resemble the micros of Krishnamurthi [14.

Several syntactic extension mechanisms have been designed for languages other than Scheme [15 16]. MacroML [17] is particularly relevant since it automatically prevents unintended variable capture. Their system is restrictive: binding forms can only extend ML's let form, and macros cannot inspect or destructure their syntactic arguments. Our work allows destructuring of S-expressions while still preserving the integrity of expressions. Our work also provides a theory of $\alpha$ equivalence. Previous work on staged notational definitions [18] provides a metalanguage SND for reasoning about MacroML programs; we believe our system more closely matches the informal reasoning used by macro programmers.

The shape types of Culpepper and Felleisen [8] are similar in expressive power to ours, allowing destructuring of S-expressions and synthesis of arbitrary binding forms. Our work extends theirs by accounting for binding structures. Crucially, this provides us with our account of $\alpha$-equivalence and hygiene. Our use of types for expressing bindings was inspired by the nominal datatypes of Gabbay and Pitts 9].

Gasbichler [19] provides a detailed formal account of a rich macro system in order to study the interaction of hygienic macros and module systems. Our work is concerned instead with the guarantees provided by hygiene. Griffin [20] and Bove and Arbilla 21 also provide formal accounts of notational definitions and macros, respectively. The former is based on a higher-order representation of binding forms, the latter on de Bruijn indices. We have taken an explicitly-named approach in order to explore the connection between hygiene and $\alpha$-equivalence. Both works prove key correctness properties, but in the context of a language with only top-level macro definitions, i.e., without lexically scoped macros.

Finally, we note that the design of our shape types bears some resemblance to the locally nameless approach to binding structures [22 2324]. In particular, our macro types use tree locations $\ell$ in order to avoid using an $\alpha$-equivalence relation on shape types, but when destructuring a type, we instantiate these locations with concrete names. We intend to investigate this relationship further.

There is much more to discover of the theory of hygienic macros. Our elementary type system is not yet expressive enough to permit important idioms in common use, including recursive macros, variable-length lists and list-patterns [25], and case dispatch. Another important next step will be to understand the type structure of higher-order macros, which expand into subsequent macro definitions. We intend to investigate the connection to staged types for this question. 
Other areas for future exploration include procedural macros, inference for shape types, and support for intentional capture.

\section{References}

1. Kiselyov, O.: How to write seemingly unhygienic and referentially opaque macros with syntax-rules. In: Scheme Workshop (2002)

2. Kohlbecker, E., Friedman, D.P., Felleisen, M., Duba, B.: Hygienic macro expansion. In: LISP and Functional Programming (1986)

3. Clinger, W., Rees, J.: Macros that work. In: POPL (1991)

4. Bawden, A., Rees, J.: Syntactic closures. In: LISP and Functional Programming, pp. 86-95 (1988)

5. Kent Dybvig, R., Bruggeman, R.H.,, C.: Syntactic abstraction in Scheme. Lisp and Symbolic Computation 5(4), 295-326 (1993)

6. van Tonder, A.: SRFI 72: Hygienic macros. Online (September 2005)

7. Clinger, W., Rees, J.: Revised ${ }^{4}$ report on the algorithmic language Scheme. Technical report (1991)

8. Culpepper, R., Felleisen, M.: Taming macros. In: Karsai, G., Visser, E. (eds.) GPCE 2004. LNCS, vol. 3286, Springer, Heidelberg (2004)

9. Gabbay, M.J., Pitts, A.M.: A new approach to abstract syntax with variable binding. Formal Aspects of Computing 13(3-5), 341-363 (2001)

10. Gorn, S.: Explicit definitions and linguistic dominoes. In: Systems and Computer Science, Proceedings of the Conference held at Univ. of Western Ontario (1967)

11. Barendregt, H.P.: The Lambda Calculus: Its Syntax and Semantics. revised edn. North-Holland, Amsterdam (1984)

12. Wright, A.K., Felleisen, M.: A syntactic approach to type soundness. Information and Computation 115(1), 38-94 (1994)

13. Flatt, M.: PLT MzScheme: Language manual. Technical Report PLT-TR2007-1v371, PLT Scheme Inc. (2007), http://www.plt-scheme.org/techreports/

14. Krishnamurthi, S.: Linguistic Reuse. PhD thesis, Rice University (May 2001)

15. de Rauglaudre, D.: Camlp4 reference manual. Online (September 2003)

16. Sheard, T., Peyton Jones, S.: Template metaprogramming for Haskell. In: Chakravarty, M.M.T. (ed.) Haskell Workshop, pp. 1-16 (2002)

17. Ganz, S.E., Sabry, A., Taha, W.: Macros as multi-stage computations: Type-safe, generative, binding macros in MacroML. In: ICFP 2001, pp. 74-85 (2001)

18. Taha, W., Johann, P.: Staged notational definitions. In: Pfenning, F., Smaragdakis, Y. (eds.) GPCE 2003. LNCS, vol. 2830, pp. 97-116. Springer, Heidelberg (2003)

19. Gasbichler, M.: Fully-parameterized, first-class modules with hygienic macros. PhD thesis, University of Tübingen (August 2006)

20. Griffin, T.: Notational definition - a formal account. In: LICS 1988, pp. 372-383 (1988)

21. Bove, A., Arbilla, L.: A confluent calculus of macro expansion and evaluation. In: LISP and Functional Programming, pp. 278-287. ACM Press, New York (1992)

22. McKinna, J., Pollack, R.: Some lambda calculus and type theory formalized. Journal of Automated Reasoning 23, 373-409 (1999)

23. Gordon, A.D.: A mechanisation of name-carrying syntax up to alpha-conversion. In: Workshop on HOL Theorem Proving and its Applications, pp. 413-425 (1994)

24. McBride, C., McKinna, J.: Functional pearl: I am not a number-I am a free variable. In: Haskell Workshop, pp. 1-9 (2004)

25. Kohlbecker, E.E., Wand, M.: Macro-by-example: Deriving syntactic transformations from their specifications. In: Principles of Programming Languages (1987) 